PPPL--2662

DE90 005803

\title{
Neoclassical Transport of Energetic Minority Tail Ions Generated by Ion-Cyclotron Resonance Heating in Tokamak Geometry
}

\author{
C.S. Chang; G.W. Hammett, and R.J. Goldston \\ Princeton Plasma Physics Laboratory \\ Princeton, NJ 08543
}

\begin{abstract}
Neoclassical transport of energetic minority tail ions, which are generated by high powered electromagnetic waves of the Ion Cyclotron Range of Frequencies (ICRF) at the fundamental harmonic resonance, is studied analytically in tokamak geometry. The effect of Coulomb collisions on the tail ion transport is investigated in the present work. The total tail ion transport will be the sum of the present collision-driven transport and the wave-driven transport, which is due to the ICRF-wave scattering of the tail particles as reported in the literature. The transport coefficients have been calculated kinetically, and it is found that the large tail ion viscosity, driven by the localized ICRF-heating and Coulomb slowing-down collisions, induces purely convective particle transport of the tail species, while the energy transport is both convective and diffusive. The rate of radial particle transport is shown to be usually small, but the rate of radial energy transport is larger and may not be negligible compared to the Coulomb slowing-down rate.
\end{abstract}

"Courant Institute of Mathematical Sciences, New York University 


\section{Introduction}

Auxiliary heating of tokamak plasmas by electromagnetic waves in the Ion Cyclotron Range of Frequencies (ICRF-waves) is becoming one of the promising schemes to heat the plasma to the temperature required for controlled thermonuclear fusion reactions. Experiments in Princeton Large Torus (PLT),' Joint European Torus (JET), ${ }^{2}$ and Toroidal Fusion Test Reactor (TFTR) ${ }^{3}$ have demonstrated that ICRF heating at fundamental harmonic resonance can generate very energetic minority tail ions (with average energy ranging over $1 \mathrm{MeV}$ in JET). Since the rate of energy transfer from the energetic tail ions to the main plasmas is proportional to the energy content in the tail ions, it is important to understand the radial confinement property, and thus the radial profile of particle and energy density, of the tail ion species.

Due to the spatially localized heating effect and highly non-Maxwellian nature of the tail distribution, the neoclassical equilibrium properties of the tail distribution are quite different from those of the background Maxwellian plasma and the resulting transport does not obey the usual neoclassical banana descriptions. Several theoretical attempts have appeared in the literature, exploring the new features of ICRF-driven non-Maxwellian transport. Reference 4 has calculated the non-Maxwellian distribution function of tail ions in the strong heating limit, and obtained the neoclassical diffusion coefficients driven by the Coulomb slowing-down collisions, predicting that the radial loss rate of the tail ions will be about one banana width in a slowing- 
down collision time. However, Ref. 4 used a bounce-averaged ICRF-heatirg operator at a place in the calculation where the local operator was needed. This made it neglect the unconventional and important effect of poloidally local heating by resonance with the ICRF waves. As will be shown in the present work, neglect of the poloidally local heating effect led Ref. 4 to describe incorrectly the basic particle transport mechanism as a diffusive process instead of a convective process.

In this work, we concentrate on the collision-driven transport of the non-Maxwellian tail ions. We will assume $k_{\|}=0$ and, hence, ignore the ICRF-driven parallel friction and transport discussed in Ref. 5, which is due to toroidal momentum input from the wave. The total transport will be the sum of the ICRF-driven transport of Ref. 5 and the collision-driven transport of the present paper. The analytic estimates in Ref. 5 led to the corclusion that RF-driven transport is usually unimportant. However, the numerical calculations of Refs. 6 and 7 show that if the RF power profile is so narrow and the tail ions become so energetic that their banana widths become a significant fraction of the RF-power profile width, then RF-driven transport can play a significant role in the central power balance of the tail ions. These numerical calculations found that for some JET cases the RF-driven transport can lead to a significant hollowing of the minority density profile, broadening the effective heating profile by a factor of $\sim 2$, and reducing the central minority energy from $\sim 1 \mathrm{MeV}$ to $\sim 100 \mathrm{keV}$. The RE power profile in JET is faily narrow, with $P(r)$ proportional to $\exp \left[-r^{2} /\left(2 \sigma^{2}\right)\right]$ and $\sigma \sim 15 \mathrm{~cm}$. By comparison, the central banana width of 
a hydrogen ion is $\sim 37 \mathrm{~cm}$ at $1 \mathrm{MeV}$, and $\sim 17 \mathrm{~cm}$ at $100 \mathrm{keV}$. The numerical calculation of Refs. 6 and 7 ignored the effects of neoclassical collision-driven transport discussed here, which may fill in the hollow minority profiles found in Refs. 6 and 7.

We study the neoclassical transport of non-Maxwellian tail ions with a quasilinear ICRF operator and Coulomb scattering operator when the minority tail-ion species is in resonance with a ICRF wave at the fundamental harmonic. We use a rigorous ordering scheme which is consistent with the repetitive local heating and cooling of the trapped tail iors, whose energy is above the ion-electron critical slowing-down energy, as they execute banaza motions. And we find that, even though the radial transport rate is roughly consistent with that given by Ref. 4, the transport mechanism is quite different: The large parallel viscosity created by the local ICRF-heating and Coulomb cooling of the tail ions induces convective radial transport for the particles, and convective and diffusive radial transport for the energy. It can be concluded from the present work that the neoclassical particle loss rate of the tail ions is usually not seriously large, of order a banana width in a slowing-down time. However, the energy transport rate can be considerably larger due to the large numerical coefficients resulting from the non-Maxwellian velocity moment of the tail distribution function. And the energetic tails can lose their kinetic energy by radial neoclassical transport mechanisms on a time scale comparable to the slowing-down time.

In Section II, a heuristic description of the basic physical mechanisms, which lead to the radial convective transport of tail ions from the single 
particle and fluid point of view, is discussed. A full kinetic calculation of both particle and energy transport is given in Section III, and some discussions are presented in Section IV. 


\section{Heuristic Description of Tail-Ion Transport Under Strong ICRF Heating}

In this section we will illustrate the basic physical mechanisms which govern the radial motion of the high energy minority ions generated by strong ICRF heating. The earlier part of the desuriptions given here is not new and is presented here for heuristic purposes to make clearer presentation of the tail-ion transport physics. During the guiding-center motion of the resonant ions, two different scattering processes compete against each other and determine the steady-state motion of the guiding center. One is the usual Coulomb scattering process relaxing the particle distribution function toward a thermal equilibrium state, and the other is the ICRFheating process ${ }^{8}$ increasing the perpendicular (to magnetic field vector $\vec{B}$ ) energy of the resonant particles as they pass through the resonance layer defined by, at the fundamental harmonic resonance

$$
\omega=\Omega_{R}+k_{\|} v_{\|}
$$

where $\omega$ is the angular frequency and $k_{\|\|}$is the parallel (to $\vec{B}$ ) wave number of a given ICRF wave, and $\Omega_{R}=e B_{R} / M_{t} c$ is the gyrofrequency of tail ions, whose mass is $M_{t}$ and charge is e, at the resonance location $B=B_{R}$. We will assume that the Doppler broadening of the resonance layer due to $k_{\|}$ is small, and thus will set $k_{\|}=0$ for the rest of the present work. For the effect of $k_{i l} \neq 0$, we refer to Ref. 5 .

Since ICRF heating increases the perpendicular energy of the resonant ions preferentially, the well-heated ions will execute banana orbits. It is easy 
to see that as the perpendicular heating at the resonance location proceeds further, the turning points of the resonance particles will asymptotically approach the resonance location where $B=B_{R}$, as was first observed experimentally. ${ }^{9}$ We can use the following simple argument to understand this phenomenon: At the resonarce location, the kinetic energy of a resonant particle can be written as $\varepsilon=M_{t} v_{\|}^{2}+\mu B_{R}$, where $\mu=M_{t} v_{\perp}^{2} / 2 B$ is the magnetic moment. Thus the increase in the particle kinetic energy $\Delta \varepsilon$ due to the perpendicular beating of the amount $\Delta \mu$ at the resonance location is given by

$$
\Delta \varepsilon=B_{R} \Delta \mu .
$$

The turning point given by $B_{t}=\varepsilon / \mu$ is redefined after the heating processes at the resonance location

$$
B_{\epsilon}=\left(\epsilon+B_{R} \Delta \mu\right) /(\mu+\Delta \mu)
$$

where Eq. (2) has been used for $\Delta \varepsilon$. In the strong heating limit, we then have

$$
B_{\imath} \rightarrow B_{R}, \text { for } \Delta_{\mu} / \varepsilon \gg 1 \text {. }
$$

Therefore, the high energy tail ions generated by high power ICRF heating execute banana orbital motions with turning points near the resonance layers. They get heated when they reach the tips of the banana orbits. but cooled during the rest of the orbital motions by Coulomb collisions (see Fig. 1). For the tail ions considered here, whose kinetic energy is much higher than the critical ion-electron slowing-down energy, the main Coulomb collisional effect is from slowing-down by background electrons. 
And the steady-state particle motion is achieved when the energy gain by ICRF heating at the turning points balances the energy loss by the slowingdown collisions.

The radial motion of our tail ions can be understood by considering the conservation of canonical angular momentum. ${ }^{10}$ The collisionless orbits are confined to surfaces of constant canonical angular momentum:

$$
R^{2} \nabla \zeta \cdot(M \vec{v}+e \vec{A} / c)=\text { constant }
$$

where $\mathrm{R}$ is the major radius, $\zeta$ is toroidal angle, $\vec{A}$ is the vector potential. The relation between the vector potential $\vec{A}$ and a radial coordinate $\psi$ ( poloidal fiux) can be identified from Faraday's law

$$
\nabla \times \vec{E}=-\frac{1}{c} \frac{\partial \vec{B}}{\partial t}
$$

where $\vec{E}$ is the electric field vector.

By multiplying $\nabla \psi$ on both sides of Eq. (4) and using the following relations

$$
\begin{gathered}
\nabla \psi=R B_{p} \dot{\psi}, \\
\hat{\psi} \cdot \nabla \times \vec{E}=\frac{\vec{B}}{R B_{p}} \cdot \nabla\left(R E_{T}\right), \\
\frac{\partial}{\partial t} \nabla \cdot(\psi \vec{B})=\frac{\partial}{\partial t} \vec{B} \cdot \nabla \psi=0,
\end{gathered}
$$

where $B_{P}\left(B_{T}\right)$ is the poloidal(toroidal) component of $\vec{B}$ and $\hat{\psi}=\nabla \psi /|\nabla \dot{\psi}|$ : we can easily change Eq. (4) into $\vec{B} \cdot \nabla\left(c R E_{T}-\partial \psi / \partial t\right)=0$, or

$$
\frac{\partial w}{\partial t}=c R E_{T}
$$


From Eq. (4) and the relation $\vec{B}=\nabla \times \vec{A}$, we also have $c E_{T}=-\partial A_{T} / \partial t$, and thus Eq. (6) becomes

$$
\psi=-R A_{T}
$$

Using this expression for the vector potential, the conservation of canonical angular momentum is expressed as

$$
\psi-c M v_{T} R / e=\text { constant }
$$

where $v_{T}$ is the toroidal component of the particle velocity.

Information on the net radial motion of the trapped particles can be obtained by taking a time derivative along the particle trajectory:

$$
\frac{d \psi}{d t}=\frac{\partial \psi}{\partial t}+\vec{v} \cdot \nabla \psi=\frac{c \dot{M}}{e} \frac{d}{d t}\left(R v_{T}\right)
$$

Integrating the above equation between two successive times $t_{1}$ and $t_{2}$, at which moments $v_{T}$ vanishes (turning points), we obtain

$$
\{\vec{v} \cdot \nabla \psi\}=-\left\{\frac{\partial \psi}{\partial t}\right\}
$$

where $\{\cdots\}$ denotes an orbital average. Since $\partial \psi / \partial t$ is related to the radial flux surface velocity $\vec{u}_{s}$ through the relation

$$
\frac{\partial \psi}{\partial t}+\vec{u}_{s} \cdot \nabla \psi=0
$$

Eq. (9) yields an interesting result

$$
\{\vec{v} \cdot \nabla \psi\}=\left\{\vec{u}_{s} \cdot \nabla \psi\right\}
$$


i.e., the average radial motion of the trapped particles is equal to the radial motion of the poloidal flux surface. According to Eq. (6), the average radial velocity can be expressed in terms of the toroidal electric field as

$$
\{\vec{v} \cdot \nabla \psi\}=-\left\{c R E_{T}\right\}
$$

which describes the inward pinch effect of banana particles first described by Ware ${ }^{11}$ and Galeev. ${ }^{12}$

As the banana particles suffer from Coulomb and RF scatterings, the radial pinch effect described above will be modified. If the Coulomb collisions are too frequent, banana orbits will not be allowed at all and the pinch effect will be absent. In the weakly collisional case, when the banana motions are well defined, the scattering effect can make the nat radial motion deviate from Eqs. (9) and (10) by disturbing $v_{T}$ and adding a friction $F_{T}$ in Eq. (8) as follows:

$$
\left.\frac{c M}{e} \frac{d\left(R v_{T}\right)}{d t}\right|_{\text {collisions }}=\frac{c R F_{T}}{e} .
$$

If there is a net toroidal torque $\left\{R F_{T}\right\}$ during a complete banana motion, we will have additional radial motions which will make the radial particle motion not to coincide with the radial flux surface motion.

The RF scattering at the resonance location, with $k_{\|}=0$, does not change toroidal momentum and thus does not contribute to the net toroidal torque. The toroidal torque from Coulomb slowing-down collisions changes signs as particle's $v_{\|}$changes sign, and thus the net torque $\left\{R F_{T}\right\}$ will not be zero if there is some asymmetry in the scattering between the positive 
and negative $v_{\|}$motions (see Fig. 2). The qualitative features of this transport mechanism were first pointed out by Ref. 14. There can be two different physical mechanisms to give rise to such asymmetry in $F_{T}$ during a banana motion: One is the difference in the path length of the particle guiding center due to the magnetic shear, and the other is the difference in the slowing-down rate $1 / T$, due to the radial gradient in the background electron density and temperature.

Radial flow due to the magnetic shear can be estimated as follows. The connection length $q R$ generally increases as the minor radius increases. Thus the path length is greater during the outer half of the banana motion than the inner half by the approximate amount, $\Delta_{b} \partial(g R) / \partial r$, where $\Delta_{b}$ is the radial banana width. Hence, the net toroidal friction integrated along a complete banana orbit takes the value

$$
\left\{F_{T}\right\} \simeq \frac{\left|v_{\|}\right|}{\tau_{s}} \frac{\Delta_{b}}{q R} \frac{\partial q R}{\partial r},
$$

where $\tau_{s}$ is the ion-electron slowing-down collision time. As a result, the banana tips will drift radially outward at the rate given by

$$
\{\vec{v} \cdot \nabla \psi\}=\frac{c R}{e}\left\{F_{T}\right\} \simeq \frac{\left|v_{\|}\right| c R}{e T_{s}} \frac{\Delta_{b}}{q R} \frac{\partial q R}{\partial r} .
$$

Radial flow of the RF-generated tail ions driven by the radial gradient in $\tau_{a}$ is estimated as follows: The net toroidal friction due to the difference in the slowing-down rate is

$$
\left\{F_{T}\right\} \simeq \frac{\left|v_{\|}\right|}{T_{s}}\left(\frac{1}{n_{e}} \frac{\partial n_{e}}{\partial r}-\frac{3}{2 T_{e}} \frac{\partial T_{e}}{\partial r}\right),
$$


and the corresponding radial drift speed is then given by

$$
\{\vec{v} \cdot \nabla \psi\} \simeq \frac{\left|v_{\|}\right| c R}{e \tau_{s}} \Delta_{b}\left(\frac{1}{n_{e}} \frac{\partial n_{e}}{\partial r}-\frac{3}{2 T_{e}} \frac{\partial T_{e}}{\partial r}\right)
$$

Summing up Eqs. (11) and (12), we obtain

$$
\{\vec{v} \cdot \nabla \psi\} \simeq \frac{\left|v_{\|}\right| c R}{e T_{s}} \Delta_{b}\left(\frac{1}{q R} \frac{\partial q R}{\partial r}+\frac{1}{n_{e}} \frac{\partial n_{e}}{\partial r}-\frac{3}{2 T_{e}} \frac{\partial T_{e}}{\partial r}\right) .
$$

Equation (13) represents the radial flow of ICRF-generated tail jons due to the collisional dissipation, in addition to the collisionless pinch effect. Notice here that the flow of particles given by Eq. (13) is all convective. This is due to the lack of randomness in the slowing-down process of the tail ions at well above the critical slowing-down energy. We note here that the same conclusion may not apply to radial heat flow, since the momentum conservation law we used here does not apply to the heat flow. This point will be addressed in detail in the next section where a full kinetic calculation is presented.

The convective transport process of tail ions described above is absent in the usual neoclassical theories. Since the usual neoclassical transport is often more easily understood with fluid equations, identification of the above simple transport mechanisms with fluid friction and viscosity may help clarify the difference in the transport processes between the usual neoclassical and present prucesses. This identification can also help understand each terms in the kinetic description presented in the next section in terms of fluid quantities. 
As a function of fluid quantities, the radial particle flux $\Gamma$ can be expressed as follows ${ }^{10}$ :

$$
\Gamma=\langle n \vec{u} \cdot \nabla \psi\rangle=-\frac{c}{\epsilon}<R^{2} \nabla \zeta \cdot(\vec{F}-\nabla \cdot \mathbf{P}+e n \vec{E})>+\mathcal{O}\left(\rho^{3}\right),
$$

where $\langle\cdots\rangle$ represents the flux-surface average, $\vec{u}$ is the fluid flow velocity, and $\vec{F}$ is the frictional force; and the quantity $\mathrm{c} / \mathrm{e}$ has been treated as first order in gyroradius $\rho$ in consideration of the $\nabla \psi$ multiplied to left-hand side.

The first term in the right-hand side of Eq. (14) is the toroidal frictional force due to the difference in the toroidal flow speed between different plasma species, and is first order in gyroradius. This term alone wili yield diffusive transport driven by the gradients in density and pressure of the tail species. The second term can be shown to correspond to an off-diagonal stress tensor term as follows:

$$
\left\langle R^{2} \nabla \zeta \cdot \nabla \cdot \mathbf{P}\right\rangle=\left\langle\nabla \cdot\left(R^{2} \nabla \zeta \cdot \mathbf{P}\right)\right\rangle=\frac{d}{d \psi}\left\langle R^{2} \nabla \zeta \cdot \mathbf{P} \cdot \nabla \psi\right\rangle .
$$

And the third term represents the toroidal electric field, which gives rise to the Ware pinch effect. For simplicity, we disregard this third term from our discussion.

For a plasma usually described in the literature, the lowest order distribution function is a Maxwellian. For such a more familiar plasma, the off-diagonal stress tensor term is of second order in gyrorarius (this point will be made clearer in the next section). Hence, the second term is small compared to the first term, and thus the neaclassical radial transport arises solely from the toroidal frictional force in the Maxwellian plasma case. 
The present problem of high energy tail transport is different from the usual one in that the lowest order distribution function is not an isotropic Maxwellian and, more importantly, in that a true local steady state is not established. Instead, the anisotropic tail distribution reaches a global, and time-averaged steady state over the bounce motions of the banana motions only. At the banana tips the particles are heated by ICRF heating, and at all other locations during their banana motions they are cooled by Coulomb slowing-down collisions. The steady condition $d f / d t=0$ is not satisfied locally, but is satisfied only over the bounce average operation, $\{d f / d t\}_{b}=0$. As a consequence, even the zeroth order (in gyroradius) distribution function gets distorted by the locally nonvanishing scattering process. Unlike the usual Maxwellian plasma, such tail plasmas generate large off-diagonal stress tensor elements which is first order in gyroradius, as will become more explicit in the next section. The off-diagonal stress tensor term, second term, in Eq. (14) is then of the same order as the first term for our tail particles and yields the convective transport terms as well as diffusive terms. Our kinetic analysis shows that the diffusive terms from the off-diagonal stress tensor exactly cancel the diffusive contribution from the friction term (first term) in Eq. (14), leaving only the convective transport from the off-diagonal stress tensor for the particle flux across the flux surfaces. 


\section{Kinetic Calculation}

We will use a well-known flux coordinate system $(\psi, \theta, \zeta)$, where $\psi$ is the enclosed poloidal flux surface and serves as a radial coordinate, $\theta$ is the poloidal angle, and $\zeta$ is the toroidal angle. The magnetic field can be expressed as

$$
\vec{B}=I(\psi) \nabla \zeta+\nabla \zeta \times \nabla \psi
$$

Parallel derivatives can be expressed as

$$
\hat{n} \cdot \nabla=\frac{1}{\mathcal{J} B} \frac{\partial}{\partial \theta},
$$

where axisymmetry is assumed so that $\partial / \partial \zeta=0$, and $\mathcal{J}=[\nabla \psi \times \nabla \theta \cdot \nabla \zeta]^{-1}$ is the Jacobian. The flux surface average of a quantity $g$ is then written as

$$
\langle g\rangle=\frac{\int d \theta \mathcal{J} g}{\int d \theta \mathcal{J}},
$$

and the bounce average is defined as

$$
\{g\}_{b}=\frac{1}{\tau_{b}} \int \frac{\mathcal{J} B}{\left|v_{j}\right|} d \theta g, \quad \tau_{b}=\int \frac{\mathcal{J} B d \theta}{\left|v_{\|}\right|},
$$

where the bounce integral is taken between $\theta=-\pi$ and $\pi$ for the passing particles and between $-\theta_{t}$ to $\hat{\theta}_{t}$ for the trapped particles, where $\pm \theta_{t}$ are the poloidal angles at the turning points where $v_{\|} \rightarrow 0$. The flux surface average of a velocity integral can be expressed in terms of the bounce average as follows:

$$
\begin{aligned}
\left\langle\int d^{3} v g\right\rangle & =\frac{1}{\int d \theta \mathcal{J}} \int d \theta \mathcal{J} 2 \pi B \sum_{\sigma} \int \frac{d E d \mu}{\left|v_{\|}\right|} g \\
& =2 \pi \sum_{\sigma} \int d E d \mu \frac{\tau_{b}}{\int \mathcal{J} d \theta}\{g\}_{b} .
\end{aligned}
$$


where $E$ is the kinetic energy, $\mu$ is the magnetic moment per unit mass, and $\sigma=\operatorname{Sign}\left(v_{\|}\right)$.

We introduce a useful identity for the Alfvén approximation of the drift velocity $\vec{v}_{d}=-v_{\|} \hat{n} \times \nabla\left(v_{\|} / \Omega\right)$, which is repeatedly used in our calculation:

$$
\vec{v}_{d} \cdot \nabla f=\frac{I v_{\|}}{\mathcal{J} B}\left[\frac{\partial}{\partial \theta}\left(\frac{v_{\|}}{\Omega}\right) \frac{\partial f}{\partial \psi}-\frac{\partial}{\partial \psi}\left(\frac{v_{\|}}{\Omega}\right) \frac{\partial f}{\partial \theta}\right]
$$

The particular usefulness of the above relation is realized when we take the bounce average of $v_{d} \cdot \nabla f$ over a banana motion. Making use of integrationby-parts and the condition $v_{\|}=0$ at the turning points, we can easily show

$$
\int \frac{d \theta \mathcal{J} B}{v_{\|}} \vec{v}_{d} \cdot \nabla f=-I \frac{\partial}{\partial \psi} \int d \theta \frac{v_{\|}}{\Omega} \frac{\partial f}{\partial \theta}
$$

A quasilinear form ${ }^{13}$ of the drift-kinetic equation can be written in the form

$$
\frac{\partial f}{\partial t}+\left(\imath_{\|} \hat{n}+\vec{v}_{d}\right) \cdot \nabla f-\frac{e}{M_{t}} E_{\|} \frac{\partial j}{\partial \imath_{\|}}=C(f)+Q(f)=C^{-}(f),
$$

where $f=f(E, \mu, \sigma, \psi, \theta, t)$ is the gyrophase-averaged value of the quasistatic part of particle distribution function, $\hat{n}=\vec{B} / B$ is the unit vector in the direction of local $\vec{B}, C(f)$ is the usual Coulomb scattering operator, and

$$
Q(f) \equiv-\nabla_{v} \cdot \Gamma_{\mu}
$$

represents the quasilinear scattering by $R F$ waves with $\Gamma_{\mu}$ defined to be the RF-driven quasilinear particle flux in velocity space. Detailed justification of Eq. (18) can be found in Ref. 4. 
In order to obtain a formal solution to Eq. (18), we use the usual small banana width ordering $\delta \equiv \Delta_{b} / a \ll 1$, where $\Delta_{b}$ is the banana width and $a$ is the plasma radius. This ordering may restrict the validity of present analyses to plasmas with larger minor radius or stronger magnetic field, since the radial excursion of the rf-produced high-energy tail ions tends to be large. For equilibrium distribution of the high energy tail ions, wherc the heating by RF waves is balanced by the collisional cooling, we adopt the usual transport ordering and take $\partial / \partial t$ to be of second order in $\delta$. The parallel electric field term in Eq. (18) has to be small for equilibrium in order to avoid massive electron runaway, and for ion species it is usually treated ${ }^{10}$ as second order in $\delta$. We expand $f$ in $\delta$,

$$
f=f_{0}+f_{1}+f_{2}+\cdots,
$$

and obtain the first two lowest order equations as follows:

$$
\begin{array}{r}
v_{\|} \hat{n} \cdot \nabla f_{0}=C\left(f_{0}\right)+Q\left(f_{0}\right)=C^{*}\left(f_{0}\right) \\
v_{\mid} \mid \hat{n} \cdot \nabla f_{1}-C^{*}\left(f_{1}\right)=-\overrightarrow{v_{d}} \cdot \nabla f_{0} .
\end{array}
$$

For the high energy tail jois the collision frequencits are inucis smaitet than the parallel transit frequency $\hat{\nu}=\left(\nu_{c}+\nu_{\mu}\right) L / v_{\|} \ll 1$, where $\nu_{c}$ is the $90^{\circ}$-Coulomb collision rate, $\nu_{\mu}$ represents the quasilinear scattering rate by RF waves, and $L$ is the scale length in the direction parallel to the magnetic field lines. To incorporate this additional smallness parameter into our analyses, we use a double ordering scheme and write $f$ as 


$$
\begin{aligned}
& f_{0}=f_{0}^{(0)}+f_{0}^{(1)}+f_{0}^{(2)}+\cdots, \\
& f_{1}=f_{1}^{(0)}+f_{1}^{(1)}+f_{1}^{(2)}+\cdots,
\end{aligned}
$$

where the superscripts represent expansion in $\hat{\nu}$. The zeroth order equation in $\delta$, Eq. (19), can then be further divided into

$$
\begin{aligned}
& v_{\|} \hat{n} \cdot \nabla f_{0}^{(0)}=0, \\
& \vartheta_{\|}^{\hat{n}} \cdot \nabla f_{0}^{(3)}=C^{-}\left(f_{0}^{(\hat{0})}\right),
\end{aligned}
$$

and the first order equation in $\delta$, Eq. (20), becomes

$$
\begin{aligned}
v_{\|} \bar{n} \cdot \nabla f_{1}^{(0)} & =-\vec{v}_{d} \cdot \nabla f_{0}^{(0)}, \\
v_{\|} \hat{n} \cdot \nabla f_{1}^{(1)}-C^{-1}\left(f_{1}^{(0)}\right) & =-\vec{v}_{d} \cdot \nabla f_{0}^{(1)},
\end{aligned}
$$

where the next order equations in $\hat{\nu}$ are omitted for simplicity.

Equation (21) is a mathematical statement of the physical fact that the dissipationless lowest order distribution function is constant along magnetic field lines. A solution for $f_{0}^{(0)}$ can be obtained by averaging Eq. (22) over the bounce motion of the trapped tail ions:

$$
\left\{v_{\|} \hat{\mathbf{n}} \cdot \nabla f_{0}^{(1)}\right\}_{b}=\left\{C^{-}\left(f_{0}^{(0)}\right)\right\}_{b}
$$

The left-hand side of Eq. (25) is identically zero because of the relation $\hat{n} \cdot \nabla=(\mathcal{J} B)^{-1}(\partial / \partial \theta)$. The function $f_{0}^{(0)}$ can be taken out of the bounce 
average operator by using Eq. (21), and thus leading to

$$
\left\{C^{*}\left(f_{0}^{(0)}\right)\right\}_{b}=\{C\}_{b}\left(f_{0}^{(0)}\right)+\{Q\}_{b}\left(f_{0}^{(0)}\right)=0 .
$$

Detailed study of this equation and the solution for $f_{0}^{(0)}$ are discussed elsewhere. ${ }^{4,16-18}$ In the present work, we will consider that $f_{0}^{(0)}$ is given in obtaining the formal description of the transport coefficients. Our final transport coefficients are not sensitive to the details of the shape of $f_{0}^{(0)}$ but depends only on the moments of $f_{0}^{(0)}$. The next order solutions $f_{0}^{(1)}, f_{1}^{(0)}$. and $f_{1}^{(1)}$ can be expressed in terms of $f_{0}^{(0)}$ using Eqs. (22), (23), and (24).

The quasilinear RF-scattering operator $Q$ can be written as

$$
Q(f)=\frac{1}{v_{\perp}} \frac{\partial}{\partial v_{\perp}} v_{\perp} D_{\perp} \delta(\Omega-\omega) \frac{\partial f}{\partial v_{\perp}}
$$

where $D_{\perp}$ is the quasilinear vclocity-space particle diffusion coefficjent ${ }^{15}$ driven by ICR $F$ waves at the resonance location, $\omega$ is the ICRF wave frequency, $\Omega$ is the gyrofrequency of the tail ions $(\Omega \propto 1 /$ major radius $)$, and the $\delta$-function ensures $Q=0$ everywhere except at a resonance point given by $\Omega=\omega$. This form of $Q$ corresponds to the $k_{l i}=0$ limit. Additional transport effects caused by $k_{\|} \neq 0$ have been discussed in Ref. 5 . Notice here that the combined scattering operator $C^{*}$, with full Coulomb operator and Eq. (27), is parity conserving in $v_{\|}$.

For the combined scattering operator $C^{*}$, two conservation laws are required for a steady state. The first is the particle conservation law:

$$
\int d^{3} v C^{*}(f)=0
$$


and the second is the flux-surface-averaged energy conservation law:

$$
\left\langle\int d^{3} v\left(m v^{2} / 2\right) C^{*}\left(f_{0}^{(0)}\right)\right\rangle=0
$$

We can easily see that Eq. (29) follows from Eqs. (15) and (26). The particle conservation law is stronger since it requires the equation to be true at every point in real space. Notice here that the flux-surface-averaged energy conservation law is required to the lowest order in $f_{0}^{(0)}$ only. It is interesting to find that $\mathrm{Eq}$. (26) yields many more general conservation equations. For any constant of motion $\zeta$, we have

$$
\left\langle\int d^{3} v \zeta C^{*}\left(f_{0}^{(0)}\right)\right\rangle=0 .
$$

When the tail ions are above the critical slowirg-dewn energy, and thus the pitch angle scattering is weak, most of the tail ions are trapped with their banana tips near the resonance layer, as discussed earlier. We will make use of this fact and ignore the small passing component of $f_{0}^{(\mathrm{C})}$. This makes the transport calculation somewhat simpler, allowing us to ignore the trapped-passing boundary. We also make use of the fact that $f_{0}^{(0)}$ is symmetric in $v_{\|}$in the trapped particle regime.

\section{A. Radial Particle Flux of Tail Ions}

The radial particle flux of the minority tail ions can be calculated from

$$
\begin{aligned}
\nabla \cdot(\hat{\psi} \Gamma) & =-\frac{1}{V^{\prime}} \frac{\partial}{\partial \psi} l^{\prime \prime} \Gamma \\
\Gamma & =\left\langle\int d ^ { 3 } v \left(\vec{v}_{d} \cdot \nabla \psi|f\rangle=\left\langle\int d^{3} v I \vec{v}_{\|} \cdot \nabla\left(\frac{v_{\|}}{\Omega}\right) f\right\rangle\right.\right. \\
& =-\left\langle\int d^{3} v I \frac{v_{\|}}{\Omega} \vec{v}_{\|} \cdot \nabla f\right\rangle,
\end{aligned}
$$


where the flux surface average operation is represented by $\langle\cdots\rangle, V^{\prime}=$ $\partial V / \partial \psi=2 \pi \int d \theta \mathcal{J}$ is the derivative of the enclosed volume, Alfvén's expression for the vertical drift is used, and the last equality is obtained by integration-by-parts along the magnetic field lines, using the velocity integration expressed in terms of the velocity coordinate $(E, \mu, \sigma)$ :

$$
\int d^{3} v=2 \pi B \sum_{\sigma} \int \frac{d E d \mu}{\left|v_{\|}\right|}
$$

Double expansion of $f$ then yields

$$
\dot{\Gamma}=-\left\langle\int d^{3} v I \frac{v_{\|}}{\Omega} \vec{v}_{\|} \cdot \nabla\left(f_{0}^{(0)}+f_{0}^{(1)}+f_{\mathrm{L}}^{(0)}+f_{1}^{(1)}+\cdots\right)\right\rangle .
$$

The first term is zero because of Eq. (21). Notice here that $f_{0}^{(0)}$ is even in $v_{\|}$ and, therefore, $f_{0}^{(1)}$ and $f_{1}^{(0)}$ are odd in $v_{\|}$, as can be seen from Eqs. (22) and (23). We can then easily see that the integral of the second and third terms in the parentheses vanish, since the integrands are odd in $v_{\|\|}$. Hence, the only contribution to particle flux is from $f_{1}^{(1)}$, which can be explained from the fact that the neoclassical radial transport is a consequence of collisional dissipation on the radial drift motions. Using Eq. (24) for $f_{1}^{(1)}$, we obtain

$$
\begin{aligned}
\Gamma & =\Gamma_{1}+\Gamma_{2}, \\
\Gamma_{1} & =-\left\langle\int d^{3} v \frac{I v_{\|}}{\Omega} C^{*}\left(f_{1}^{(0)}\right)\right\rangle, \\
\Gamma_{2} & =\left\langle\int d^{3} v \frac{I v_{\|}}{\Omega} \vec{v}_{d} \cdot \nabla f_{0}^{(1)}\right\rangle .
\end{aligned}
$$

Determining $f_{1}^{(0)}$ and $f_{0}^{(1)}$ in terms of $f_{0}^{(0)}$ and performing the velocity-space integral and flux-surface average vill give us the neoclassical radial particle flux of the high energy tail ions. 
We note here that the $\Gamma_{1}$ term in Eq. (31) represents the usual contribution to the neoclassical radial particle diffusion from the parallel friction force [see Eq. (14)]. We will show that this diffusive term cancels out with the diffusive part of $\Gamma_{2}$ and thus the usual banana diffusion process is absent here. The $\Gamma_{2}$ term, however, represents an unusual feature of the present problem. This term represents the off-diagonal stress tensor contribution to the neoclassical radial particle flux coming from the nonvanishing $f_{0}^{(1)}$, which does not exist to this order in the usual thermal equilibrium problem where the lowest order distribution function is a Maxwellian [see Eq. (14)]. This can be easily understood from Eq. (22), by noticing that $C\left(f_{\mathrm{o}}^{(0)}\right)$ vanishes for a Maxwellian $f_{0}^{(0)}$. The existence of nonvanishing $f_{0}^{(1)}$ is due to the localized heating operator $Q$. As discussed in the previous section, a true equilibrium distribution function satisfying $C^{*}\left(f_{0}^{(0)}\right)=0$ does not exist for our high energy tail ions, even to the lowest order in gyroradius. And the collisional steady state exists only over the bounce average orbits through Eq. (26). A high energy tail ion in resonance with $R F$ waves is heated near the turning points of the banana orbits, and loses energy during the rest of the banana motions through Coulomb collisions with the cold background particles (see Fig. 1):

$$
\begin{array}{ll}
\int d^{3} v E C^{*}\left(f_{0}^{(0)}\right)>0 & \text { at resonance surfaces, } \\
\int d^{3} v E C^{*}\left(f_{0}^{(0)}\right)<0 & \text { otherwise. }
\end{array}
$$

The first term in the expression for the radial particle flux can readily be evaluated by solving for $f_{\mathrm{l}}^{(0)}$ from Eq. (23), as in the tusual banana transport 
theories. ${ }^{10}$ Using the relation

$$
\vec{v}_{d} \cdot \nabla \psi=I v_{\|} \hat{n} \cdot \nabla \frac{v_{\|}}{\Omega}
$$

we can easily find

$$
f_{1}^{(0)}=-\frac{I v_{\|}}{\Omega} \frac{\partial f_{0}^{(0)}}{\partial \psi}+G
$$

Here $G$ is a function constant along $\vec{B}$, and can be determined from the bounce average of the next order equation, Eq. (24). The bounce avernge of the first term in Eq. (24) is trivially zero. The right-hand side of Eq. (24) is odd in $v_{\|}$since $f_{0}^{(1)}$ is an odd function of $v_{\|}$, and thus it vanishes when bounce averaged. The solubility condition for Eq. (24) then simply becomes

$$
\left\{C^{*}\left(f_{1}^{(0)}\right)\right\}_{b}=0,
$$

which is identical to the solubility condition for the usual neoclassical banana transport for thermal particles. The combined collision operator $C^{*}$ conserves parity in $\sigma=v_{\|} /\left|v_{\|}\right|$, as discussed after Eq. (27). The first term in Eq. (34) is odd in $v_{\|}$and it vanishes in the bounce-average integral of Eq. (35). $\mathrm{G}$ is independent of $\theta$ and thus it can be taken outside of the bounce integral, and Eq. (35) becomes

$$
\left\{C^{*}\right\}_{6} G=0
$$

i.e., $G$ and $f_{0}^{(0)}$ are solutions of the same equation[ see Eq. (26)]. Without loss of generality, we can absorb $G$ into $f_{0}^{(0)}$. In any case, $G$ is even in $v_{\|}$ and thus gives no contribution to the radial fluxes. Therefore, our solution for $f_{1}^{(0)}$ simply becomes

$$
f_{i}^{(0)}=-I \frac{v_{11}}{\Omega} \frac{\partial f_{0}^{(0)}}{\partial \psi}
$$


In order to be able to actually evaluate the phase-space integral to obtain the final expression for the diffusion coefficients, a simplified Coulomb collision operator is used. We assume that most of the minority tail ions have energies higher than the critical slowing-down energy above which the tail ion-electron energy transfer rate is greater than the tail ion-ion energy transfer rate. This assumption will set a lower bound to the RF-power level to ensure the validity of our analysis as discussed in Ref. 4. Then the Coulomb scattering operator for the tail ions can be written as the combination of the tail ion-electron slowing-cuwn part $C_{t e}^{s}$ and the tail ion-main ion pitch angle scattering part $C_{t i}^{\&}$, where $\xi$ is cosine of the pitch angle $\xi=v_{\|} / v$, and the subscripts $t, i$, and $e$ denote tail ion, main ion, and electron species, respectively;

$$
\begin{aligned}
C(f) & =C_{t e}^{s}+C_{t i}^{\xi}, \\
C_{t e}^{s}(f) & =\nu_{s} \frac{\partial}{\partial \vec{v}} \cdot \vec{v} f, \\
C_{t i}^{\xi}(f) & =\frac{\nu_{t i}}{2} \frac{\partial}{\partial \xi}\left(1-\xi^{2}\right) \frac{\partial f}{\partial \xi}, \\
\nu_{s} & =\frac{1}{\tau_{s}}=\frac{M_{e} n_{e}}{M_{t} n_{t} \tau_{e t}}, \\
\nu_{t i} & =\left(\frac{v_{e f J}}{v}\right)^{3} \frac{1}{\tau_{t i}} \\
\tau_{e t} & =\frac{3 \sqrt{2}}{8 \sqrt{\pi}} \frac{\sqrt{M_{e}} T_{e}^{3 / 2}}{Z_{t}^{2} e^{4} n_{t} \ln \Lambda}, \\
\tau_{t i} & =\frac{1}{4 \pi} \frac{M_{t}^{2} v_{e f f}^{3}}{Z_{i}^{2} Z_{t}^{2} e^{n_{1} n_{i} \ln \Lambda},}
\end{aligned}
$$

where $n_{t}$ is the flux-surface-averaged tail-ion density, and $v_{e f f}$ is a typical tail-ion velocity (we define $v_{e f /}$ so that $(3 / 2) n_{t} M_{t} v_{e / f}^{2}$ is the flux-surface- 
averaged tril-ion energy density). The weak pitch angle scattering is retained here because the $\partial / \partial \xi$ may be large due to the localization near the resonance pitch angle. This localization width of $f_{0}^{(0)}$ in pitch angle itself is determined from pitch angle scattering. ${ }^{4,16,18}$ But it turns out that the pitch angle scatterings make little contribution to our final evaluation of the transport coefficients, due to the fact that $\nu_{t i} \ll \nu_{s}$.

The first part in the particle flux, $\Gamma_{1}$, can 1. ow be easily evaluated to be

$$
\begin{aligned}
\Gamma_{1} & =-\left\langle\int d^{3} v I \frac{v_{\|}}{\Omega}(C+Q)\left(f_{1}^{(0)}\right)\right\rangle \\
& =\left\langle\int d^{3} v I \frac{v_{\|}}{\Omega} C\left(I \frac{v_{\|}}{\Omega} \frac{\partial f_{0}^{(0)}}{\partial \psi}\right)\right\rangle \\
& =-\left\langle\int d^{3} v\left(\frac{I v_{\| l}}{\Omega}\right)^{2}\left(\nu_{s}+\nu_{t i}\right) \frac{\partial f_{0}^{(0)}}{\partial \psi}\right)
\end{aligned}
$$

where the contribution from the RF heating operator, $Q$, has been dropped because $\mathcal{Q}$ commutes with $v_{\|}$and $Q$ conserves particle number $\left[\int d^{3} v Q(f)=\right.$ 0 for any $f]$.

The second part in the particle flux, $\Gamma_{2}$, is easily calculated by using Eq (16) for the vertical drift:

$$
\begin{aligned}
\Gamma_{2} & =\left\langle\int d^{3} v \frac{I v_{\|}}{\Omega} \vec{v}_{d} \cdot \nabla f_{0}^{(1)}\right\rangle \\
& =-\frac{2 \pi \sum_{\sigma} \sigma}{\int d \theta \mathcal{J}} \int d \theta \mathcal{J} \int d E d \mu \frac{\Gamma^{2} v_{\|}}{\mathcal{J} \Omega}\left[\frac{\partial}{\partial \psi}\left(\frac{v_{\|}}{\Omega}\right) \frac{\partial f_{0}^{(1)}}{\partial \theta}-\frac{\partial}{\partial \theta}\left(\frac{v_{\|}}{\Omega}\right) \frac{\partial f_{0}^{(1)}}{\partial i^{i}}\right] .
\end{aligned}
$$

Integrating by parts and collecting term, it is straightforward to obtain

$$
\Gamma_{2}=-\frac{2 \pi \sum_{\sigma} \sigma}{\int d \theta \mathcal{J}} \int d E d \mu I^{2} \frac{\partial}{\partial \psi^{\varphi}}\left[\int d \theta \frac{1}{2} \frac{v_{\| l}^{2}}{\Omega^{2}} \frac{\partial f_{0}^{1}}{\partial \theta}\right] .
$$


We use Eq. (22) to express $f_{0}^{(1)}$ in terms of $f_{0}^{(0)}$,

$$
v_{\|} \frac{\partial f_{0}^{(1)}}{\partial \theta}=\mathcal{J} B C^{*}\left(f_{0}^{(0)}\right)
$$

and substitute it into the above expression for $\Gamma_{2}$ to obtain

$$
\begin{aligned}
\Gamma_{2} & =-\frac{I^{2}}{\int d \theta \mathcal{J}} \frac{\partial}{\partial \psi}\left[\int d \theta \mathcal{J} \int d^{3} v \frac{1}{2} \frac{v_{\|}^{2}}{\Omega^{2}} C^{\bullet}\left(f_{0}^{(0)}\right)\right] . \\
& =-\frac{I^{2}}{\int d \theta \mathcal{J}} \frac{\partial}{\partial \psi}\left[\int d \theta \mathcal{J} \int d^{3} v \frac{1}{2} \frac{v_{\|}^{2}}{\Omega^{2}} C\left(f_{0}^{(0)}\right)\right] \\
& =\frac{I^{2}}{\int d \theta \mathcal{J}} \frac{\partial}{\partial \psi}\left[\int d \theta \mathcal{J} \int d^{3} v f_{0}^{(0)}\left(\frac{v_{\|}^{2}}{\Omega^{2}} t_{s}-\frac{1}{2} \frac{v^{2}-3 v_{\|}^{2}}{\Omega^{2}} \nu_{t i}\right)\right] .
\end{aligned}
$$

Here in the second equality, as in the case of $\Gamma_{1}$, the contribution from $Q$ vanishes because $\eta_{\|}$commutes with the heating operator $Q$ and $Q$ conserves particles.

We explicitly evaluate the $\psi$ derivative, using $\mathcal{J}=q R^{2} / I$, and assuming $I=R B_{T} \simeq$ constant. Considering the $\psi$ dependence of $\mathcal{J}, \nu_{s}, q R^{2}$, and $1 / \Omega^{2}$, the term proportional to $\nu_{s}$ in Eq. (41) is changed into

$$
\begin{aligned}
\Gamma_{2 s}= & \left\langle\int d ^ { 3 } v f _ { 0 } ^ { ( 0 ) } \frac { I ^ { 2 } v _ { \| } ^ { 2 } } { \Omega ^ { 2 } } \nu _ { s } \left(\frac{1}{f_{0}^{(0)}} \frac{\partial f_{0}^{(0)}}{\partial \psi}+\frac{1}{\nu_{s}} \frac{\nu_{s}}{\partial \psi}+\frac{1}{q R} \frac{\partial q R}{\partial \psi}\right.\right. \\
& \left.\left.+\frac{1}{\left|v_{\|}\right|} \frac{\partial\left|v_{\|}\right|}{\partial \psi}-\frac{2}{B} \frac{\partial B}{\partial \psi}\right)\right\rangle .
\end{aligned}
$$

The first term in $\Gamma_{2}$, Eq. (42), has the form of diffusion, but it is exactly equal and opposite to the component of $\Gamma_{1}$ proportional to $\nu_{s}$. Thus there is no diffusive term in $\Gamma$ proportional to $\nu_{s}$. The only diffusion is due to pitch angle scattering terms which are proportional to $\nu_{t i}$. The other terms in Eq. (42) represent convective transport due to the asymmetric 
banana-orbital effects between the radially inside and outside half of the banana motions, as discussed in Sec. II. For example, the second term in Eq. (42) is driven by the asymmetry due to the radial gradient in the slowing-down collision frequency; the third term is driven by the magnetic shear, or equivalently by the asymmetry in the orbital path lengths between the inside and outside banana orbits; and the fourth and fifth terms are driven by the asymmetry in the parallel particle velocity and magnetic field strength, respectively. Actually, the fourth and fifth terms are from the same physical origin $(\partial B / \partial \psi)$, but the fifth term is of one order higher in inverse-aspect-ratio than the rest and can be neglected. Making the large-aspect-ratio approximation, using the relation

$$
\frac{1}{\nu_{s}} \frac{\partial \nu_{s}}{\partial \psi}=\frac{1}{n_{e}} \frac{\partial n_{e}}{\partial \psi}-\frac{3}{2 T_{e}} \frac{\partial T_{e}}{\partial \psi}
$$

and summing $\Gamma_{1}$ and $\Gamma_{2}$ together, we find that the component $\Gamma_{s}$ of particle flux $\Gamma$ proportional to the slowing-down rate is given by

$$
\begin{aligned}
\Gamma_{s} & =\frac{I^{2}}{\tau_{s}}\left\langle\int d^{3} v \frac{v_{I I}^{2}}{\Omega^{2}} f_{0}^{(0)}\left[\frac{1}{n_{e}} \frac{\partial n_{e}}{\partial \psi}-\frac{3}{2 T_{e}} \frac{\partial T_{e}}{\partial \psi}+\frac{1}{q R} \frac{\partial q R}{\partial \psi}\right]\right\rangle \\
& -\frac{I^{2}}{2 \tau_{s}}\left\langle\int d^{3} v \frac{v_{1}^{2}}{\Omega^{2}} f_{0}^{(0)} \frac{1}{B} \frac{\partial B}{\partial \psi}\right\rangle .
\end{aligned}
$$

Here in the last term, which is from $\partial v_{\|} / \partial \psi$-term, the driving term $\partial B / \partial \psi_{r}$ is smaller than the other driving terms by one order in inverse aspect ratio. but it is multiplied by $v_{\perp}^{2}$ which is larger than $v_{\|}^{2}$ by one order in inverse aspect ratio.

$\Gamma_{t i}$, the part of $\Gamma$ proportional to $1 / \tau_{t i}$, is calculated in the same way as for $\Gamma_{s}$. Making the large aspect ratio approximation and thus neglecting $\iota_{\overline{\mid}}^{2}$ 
compared to $v^{2}$, we obtain

$$
\Gamma_{t i}=-\frac{I^{2}}{\int d \theta} \overline{\mathcal{J}} \frac{\partial}{\partial \psi}\left[\int d \theta \mathcal{J} \int d^{3} v f_{0}^{(0)} \frac{1}{2} \frac{v^{2}}{\Omega^{2}} \nu_{t i}\right] .
$$

We note here that if one desires to be more accurate and keep $v_{\|}^{2}$ (compared to $v^{2}$ ) in the pitch angle scattering term, one should also keep the ion-jon energy scattering effect in the Coulomb collision operator. The total flux is then given by the sum of the slowing-down contribution, Eq. (43), and the pitch angle contribution, Eq. (44): $\Gamma=\Gamma_{s}+\Gamma_{t i}$.

In order to make further progress, we need to evaluate explicitly the flux-surface-averaged velocity space moments which appear in Eqs. (43) and (44). We will evaluate these moments using a model solution for $f_{0}^{(0)}$, which is approximately valjd in the high energy limit, where the minority jons collide primarily with electrons instead of the thermal ions, and the $\mathrm{RF}$-driven velocity diffusion is balanced by drag on the electrons:

$$
\begin{aligned}
f_{0}^{(0)} & =f_{m}(E) A \delta\left(\frac{\mu}{E}-\frac{1}{B_{R}}\right), \\
f_{m}(E) & =\pi_{t}\left(\frac{M_{t}}{2 \pi T_{e f f}}\right)^{3 / 2} e^{-M_{t} E / T_{e f s}}, \\
A & =\frac{2 \int_{-.}^{\pi} d \theta \mathcal{J}}{\int_{-\theta_{R}}^{\theta_{R}} d \theta \mathcal{J} B / \sqrt{1-B / B_{R}}}, \\
T_{E f f} & =\frac{P_{r f} \tau_{s}}{3 n_{t}} .
\end{aligned}
$$

where $B_{R}$ is the magnetic field at resonance, satisfying $\omega^{\prime}=\Omega_{R}=Z_{t} \in B_{R} / M I_{t} c$. $A$ is a coefficient necessary to make $n_{t}$ the flux-surface-averaged minority tail density

$$
\left\langle\int d^{3} v f_{0}^{(0)}\right\rangle=2 \pi \sum_{\sigma} \int_{0}^{\infty} d E \int_{0}^{\infty} d \mu f_{0}^{(0)} \frac{f_{-\theta_{x}}^{\theta_{2}} d \theta \mathcal{J} B /\left|v_{\|}\right|}{\int d \theta \mathcal{J}}
$$




$$
\begin{aligned}
& =4 \sqrt{2} \pi \int_{0}^{\infty} d E \sqrt{E} f(E) \\
& =n_{t},
\end{aligned}
$$

and $\pm \theta_{R}$ are defined as the poloidal angles of the turning points which are identical to the poloidal angles of the ICRF-resonance points in the present $\delta$-function approximation of the pitch angle spread. Notice here that $f_{m}$ is the flux-surface- and pitch-angle-averaged particle distribution function, and is the same as the isotropic solution of Ref. 8 in the high energy limit where RF heating is balanced by drag due to collisions with electrons. The flux-surface-averaged energy density for our model solution is just

$$
\left\langle\int d^{3} v f_{0}^{(0)} M_{t} E\right\rangle=\frac{3}{2} n_{t} T_{e f J}
$$

The normalization coefficient $A$ can be expressed in terms of geometric constants. Using $\mathcal{J}=q R^{2} / I, A$ can be wrjtten as

$$
A=\frac{2 \int_{-\pi}^{\pi} d \theta R^{2}}{\int_{-\theta_{R}}^{\theta_{R}} d \theta R^{2} B\left(1-B / B_{R}\right)^{-1 / 2}} .
$$

Working to lowest order in inverse-aspect ratio with circular flux surfaces $R=R_{0}+r \cos \theta$ and $B=B_{0} R_{0} / R$, this can be written in terms of the complete elliptic integral $K(m)$ :

$$
A=\frac{\pi \sqrt{2 r / R_{0}}}{B_{0} K\left(\sin ^{2}\left(\theta_{R} / 2\right)\right)}
$$

If the resonance layer is near the low field side of the flux surface so that $\theta_{R} \ll 1$, then $\pi \approx \pi / 2$. 
The $\delta$-function $\mu$ dependence means that all of the minority tail ions have their banana tips exactly in the resonance layer. In reality, $f_{0}^{(0)}$ will have a finite width in $\mu$ rather than this $\delta$-function dependence. But since we only need a few velocity-space moments of $f_{0}^{(0)}$, it is not necessary to be highly accurate in the specification of $f_{0}^{(0)}$. The peaking of $f_{0}^{(0)}$ around $\mu / E=1 / B_{R}$ can be seen from the numerical solution of Eq. (26) in Refs. 16 and 17, and from the experimental observations of Ref. 9. An analytic solution for $f_{0}^{(0)}$, which includes the finite width in $\mu$ rather than a $\delta$ function, has been derived in Ref. 18.

In Eq. (44), we need to evaluate the moment

$$
\left\langle\int d^{3} v f_{0}^{(0)} \frac{v^{2}}{\Omega^{2}} \frac{\nu_{t i}}{2}\right\rangle \simeq \frac{1}{\sqrt{2 \pi}} \frac{n_{t}}{T_{t i}} \frac{v_{e f f}^{2}}{\Omega_{0}^{2}},
$$

where $v_{\text {eff }}=\sqrt{T_{\text {eff }} / M_{t}}$, and we have ignored the variation of $\Omega$ with poloidal angle to be consistent with the large-aspect-ratio approximations we used to derive Eqs. (43) and (44). We make the same approximations to evaluate the moments needed for Eq. (43):

$$
\left\langle\int d^{3} v f_{0}^{(0)} \frac{v_{\|}^{2}}{\Omega^{2}}\right\rangle \simeq 3 n_{t} \frac{v_{e f f}^{2}}{\Omega_{0}^{2}}\left\{\frac{B_{R}-B}{B_{R}}\right\}_{b},
$$

where $\{\cdots\}_{6}$ is now the bounce-averaging operator for orbits with their banana tips in the resonance layer:

$$
\left\{\frac{B_{R}-B}{B_{R}}\right\}_{b}=\frac{\int_{-\theta_{R}}^{\theta_{R}} \mathcal{J} B\left(\frac{B_{R}-B}{B_{R}}\right)^{1 / 2}}{\int_{-\theta_{R}}^{\theta_{R}} \mathcal{J} B\left(\frac{B_{R}-B}{B_{R}}\right)^{-1 / 2}} .
$$

The other moment needed for Eq. (43) is

$$
\frac{1}{2}\left\langle\int d^{3} v v_{s} f_{0}^{(0)} \frac{I^{2} v_{\perp}^{2}}{\Omega^{2}} \frac{\partial \ln B}{\partial \psi}\right\rangle \simeq \frac{1}{2 \tau_{s}}\left\langle\int d^{3} v f_{0}^{(0)} v^{2} \frac{\partial \ln B}{\partial \psi}\right\rangle \frac{I^{2}}{\Omega_{0}^{2}}
$$




$$
\simeq \frac{3}{2 \tau_{g}} n_{t}\left(\frac{I v_{e f f}}{\Omega_{0}}\right)^{2}\left\{\frac{\partial \ln B}{\partial \psi}\right\}_{b}
$$

Using the above expressions for the velocity space moments, we can write the total particle flux, which is the sum of Eqs. (43) and (44), as

$$
\begin{aligned}
\Gamma= & \Gamma_{t}+\Gamma_{t i}, \\
\Gamma_{s}= & -\frac{3 n_{t}}{\tau_{s}}\left(\frac{I v_{e f f}}{\Omega_{0}}\right)^{2}\left\{\frac{B_{R}-B}{B_{R}}\right\}_{b}\left(\frac{3}{2} \frac{\partial \ln T_{e}}{\partial \psi}-\frac{\partial \ln n_{e}}{\partial \psi}-\frac{\partial \ln q}{\partial \psi}\right) \\
& -\frac{3}{2} \frac{n_{t}}{\tau_{s}}\left(\frac{I v_{e f f}}{\Omega_{0}}\right)^{2}\left\{\frac{\partial \ln B}{\partial \psi}\right\}_{b}, \\
\Gamma_{t i}= & -\frac{I^{2}}{V^{\prime}} \frac{\partial}{\partial \psi}\left(V \cdot \sqrt{\frac{1}{2 \pi}} \frac{n_{t}}{\tau_{t i}} \frac{v_{e f f}^{2}}{\Omega_{0}^{2}}\right) .
\end{aligned}
$$

Continuing to work to the lowest order in inverse-aspect ratio, we approximate

$$
\frac{1}{V^{\prime}} \frac{\partial}{\partial \psi} V^{\prime}=\frac{1}{V^{\prime}} \frac{\partial}{\partial \psi} 2 \pi \int d \theta \frac{q R^{2}}{I} \simeq \frac{1}{q} \frac{\partial q}{\partial \psi} .
$$

Explicitly carrying out the $\psi$-derivative in $\Gamma_{t i}$, with the help of the above approximation, we get

$$
\Gamma_{t i}=-\frac{1}{\sqrt{2 \pi}} \frac{n_{t}}{\tau_{t i}}\left(\frac{I v_{e f f}}{\Omega_{0}}\right)^{2}\left(\frac{\partial \ln n_{t}}{\partial \psi}-\frac{1}{2} \frac{\partial \ln T_{e f f}}{\partial \psi}+\frac{\partial \ln n_{i}}{\partial \psi}+\frac{\partial \ln q}{\partial \psi}\right)
$$

$\Gamma_{3}$, the component of $\Gamma$ which is proportional to $1 / \tau_{3}$, has a similar magnitude as the particle flux found in Ref. 4, but has a quite different plysical meaning since Ref. 4 had a purely diffusive particle flux while the present $\Gamma$, is purely convective. This difference is due to the use of an overly simplified RF heating operator in Ref. 4. In the slowing-down contribution $\Gamma_{s}$, the electron-density-gradient-driven term is radially inward. while the 
other terms drive particles radially outward. In the pitch angle contribution $\Gamma_{t i}$, the convective terms are small compared to $\Gamma_{s}$ and are neglected to be consistent with the present approximation. And thus $\Gamma_{t i}$ is diffusive to the lowest order and may have some, if any, significance. In the usual case where $\partial n_{t} / \partial \psi<0$ and $\partial T_{\text {eff }} / \partial \psi<0$, the tail density gradient drives tail ions outward, while the tail temperature gradient drives them inwards.

We shall make some more remarks on the diffusive particle flux. $\Gamma_{t i}$, being proportional to the pitch angle scattering rate $1 / \tau_{t i}$, is the traditional drive of neoclassical banana transport and leads to a diffusive component in the particle flux. However, the dependence of the aspect ratio is not traditional. This is because we have assumed that all of the minority ions are trapped due to the perpendicular heating from ICRF waves, while in the usual case where $f_{0}^{(0)}$ is isotropic, only a small fraction, $\sim \sqrt{r / R}$, of the particles are trapped. This assumption is valid only if the minority ions are sufficiently energetic so that the pitch angle scattering rate is sufficiently small compared to the slowing-down rate. Analytic and numerical solutions for $f_{0}^{(0)}$ indicate ${ }^{4,16}$ that this requires $T_{\text {eff }}>(R / r) E_{\text {crit, }}$ where $E_{\text {crit }}$ is the critical energy above which collisions with electrons exceed collisions with jons. If all of the gradients are of the same order

$$
\frac{\partial \ln T_{e}}{\partial \psi} \sim \frac{\partial \ln n_{\varepsilon}}{\partial \psi} \sim \frac{\partial \ln n_{t}}{\partial \psi} \sim \frac{\partial \ln T_{e f f}}{\partial \psi} \sim \frac{\partial \ln q}{\partial \psi},
$$

then $\Gamma_{t i}$ will be small compared to $\Gamma_{s}$ in the high energy regime where our expressions for the particle flux are correct. We have retained the $\Gamma_{t i}$ component, however, because of its relationship to 'he usual neoclassical 
transport, because it alone contains a diffusive component, and because $\partial \ln T_{e f f} / \partial \psi$ may be very large compared to other gradients in cases where the ICRF power is highly focussed near the axis (since $T_{e / f} \propto P_{R F}$ ) while the background pressure is relatively flat.

\section{B. Radial Energy Transport of Tail Ions}

The radial energy flux calculation is similar in many ways to the particle flux calculation, so we will leave out most of the intermediate steps. The radial energy flux in the present work is defined as

$$
\begin{aligned}
\frac{\mathcal{Q}}{T_{e f f}} & =\frac{1}{2}\left\langle\int d^{3} v\left(\frac{v}{v_{e f f}}\right)^{2}\left(\vec{v}_{d} \cdot \nabla \psi\right) f_{1}^{(1)}\right\rangle, \\
& =-\frac{1}{2}\left\langle\int d^{3} v\left(\frac{v}{v_{e f f}}\right)^{2} \frac{I v_{\|}}{\Omega} \vec{v}_{\|} \cdot \nabla f_{1}^{(1)}\right\rangle
\end{aligned}
$$

where $\mathcal{Q}$ is defined in such a way that the energy loss from a flux volume is expressed as

$$
\nabla \cdot(\hat{\psi} \mathcal{Q})=\frac{1}{V^{\prime}} \frac{\partial}{\partial \psi}\left(V^{\prime} \mathcal{Q}\right)
$$

and the sole survival of $f_{1}^{(1)}$ in the expansion of $f$ is as explained in the particle flux calculation.

As in the particle flux calculation, we divide $\mathcal{Q}$ into two parts using Eq. (24),

$$
\begin{aligned}
\frac{\mathcal{Q}}{T_{e f f}} & =\frac{\mathcal{Q}_{1}}{T_{e f f}}+\frac{\mathcal{Q}_{2}}{T_{e f f}} \\
\frac{\mathcal{Q}_{1}}{T_{e f J}} & =-\frac{1}{2}\left\langle\int d^{3} v\left(\frac{v}{v_{e f f}}\right)^{2} \frac{I v_{\|}}{\Omega} C^{*}\left(f_{1}^{0}\right)\right\rangle,
\end{aligned}
$$




$$
\frac{\mathcal{Q}_{2}}{T_{e f f}}=\frac{1}{2}\left\langle\int d^{3} v\left(\frac{v}{v_{e f J}}\right)^{2} \frac{I v_{\|}}{\Omega} \vec{v}_{d} \cdot \nabla f_{0}^{(1)}\right\rangle
$$

The perturbed function $f_{1}^{(0)}$ in the expression for $\mathcal{Q}_{1}$ is easily changed into $f_{0}^{(0)}$ by using Eq. (36). In the expression for $\mathcal{Q}_{2}$, following exactly the same procedure as for $\Gamma_{2}$, we use Eq. (16) and integration-by-parts in $\theta$ to express $\vec{v}_{d} \cdot \nabla f_{0}^{(1)}$ in terms of its parallel derivative $\hat{n} \cdot \nabla f_{0}^{(1)}$. Using Eqs. (22) and (36) for $f_{0}^{(1)}$ and $f_{1}^{(0)}$, we can then express $\mathcal{Q}_{1}$ and $\mathcal{Q}_{2}$ in terms of $f_{0}^{(0)}$ as follows:

$$
\begin{aligned}
\frac{\mathcal{Q}_{1}}{T_{e f S}} & =\frac{1}{2}\left\langle\int d^{\beta} v\left(\frac{v}{v_{e S S}}\right)^{2} \frac{I v_{\|}}{\Omega} C^{*}\left(\frac{I v_{\|}}{\Omega} \frac{\partial f_{0}^{(0)}}{\partial \psi}\right)\right\rangle \\
\frac{\mathcal{Q}_{2}}{T_{e S J}} & =-\frac{1}{4 v_{e j S}^{2}} \frac{1}{V^{\prime}} \frac{\partial}{\partial \psi}\left[V^{\prime}\left\langle\int d^{\beta} v v^{2} \frac{I^{2} v_{\|}^{2}}{\Omega^{2}} C^{*}\left(f_{0}^{(0)}\right)\right\rangle\right] .
\end{aligned}
$$

The direct $\mathrm{RF}$ contribution to the heat flux through the $\mathrm{RF}$ quasilinear operator $Q$ (in $C^{*}=C+Q$ ) no longer vanishes exactly for general $f_{0}^{(0)}$ as it did in the case of the particle flux. However, due to the fact that $Q=0$ everywhere except at resonance and $v_{\|}^{2} / v^{2} \ll 1$ at resonance, the $Q$ contribution will be ignored compared to the $C$ contribution.

The collision operator in the above equations can be simplified by integrationby-parts, yielding

$$
\begin{aligned}
\frac{\mathcal{Q}_{1}}{T_{e f f}} & =-\frac{1}{2}\left\langle\int d^{3} v\left(\frac{v}{v_{e f f}}\right)^{2}\left(\frac{I v_{\|}}{\Omega}\right)^{2}\left(3 \nu_{s}+\nu_{t i}\right) \frac{\partial f_{0}^{(0)}}{\partial \psi}\right\rangle, \\
\frac{\mathcal{Q}_{2}}{T_{e f f}} & =\frac{1}{v_{e f f}^{2} V^{\prime}} \frac{\partial}{\partial \psi}\left[V^{\prime}\left\langle\int d^{3} v v^{2}\left(\frac{I}{\Omega}\right)^{2} f_{0}^{(0)}\left(\nu_{s} v_{\|}^{2}-\frac{1}{4} \nu_{t i} v^{2}\right)\right\rangle\right],
\end{aligned}
$$

where the pitch angle scattering frequency is smaller than the slowingdown scattering frequency by approximately an inverse-aspect ratio. but $\nu_{s} v_{\|}^{2} \sim \nu_{t i} v^{2}$ as explained earlier. 
Notice here that the diffusive term in $\mathcal{Q}_{2}$ proportional to the slowingdown rate (the component of $\mathcal{Q}_{2}$ proportional to $\nu_{3} \partial f_{0}^{(0)} / \partial \psi$ ) does not cancel $\mathcal{Q}_{1}$ exactly, as seen in the particle flux case. Thus, unlike the particle flux, we will have a difusive radial transport of tail-ion energy due to the slowing-down collisions. The difference between the radial particle and energy fluxes is in that there is no particle source in the velocity space scattering operator, but there is a strong energy source in the RF-scattering operator and a strong energy sink in the slowing-down operator. The mjnority tail ions absorb power from the RF waves on one flux surface, and give power to electrons (via slowing-down collisions) on other flux surfaces during banana motions (see Fig. 1). This finite banana-width spreading of the $R F$ power is represented by the diffusive terms in $\mathcal{Q}$ proportional to the slowing-down rate.

In the particle fiux calculation, the pitch-angle-scattering contribution is kept, even though $\nu_{t i} \ll \nu$, is true. This is because it contained the only diffusive transport process. However, in the case of the energy transport. the bigger slowing-down scattering contribution contains diffusive terms, and thus, keeping the higher-order diffusive terms from the pitch-anglescattering contribution is meaningless in the present situation, where the minority tail ions are so energetic that $\nu_{t i} / \nu_{s}<r / R$ is true.

Rather than explicitly evaluating $\partial f_{0}^{(0)} / \partial \psi$ in $\mathcal{Q}_{1}$, it is convenient to interchange the order of $\psi$-derivative so that only integrals over $f_{0}^{(0)}$ are 
needed, i.e.,

$$
\begin{aligned}
\frac{\mathcal{Q}_{1}}{T_{e f f}}= & -\frac{3}{2 v_{e f f}^{2}} \frac{1}{\int d \theta \mathcal{J}} 2 \pi \sum_{a} \int d E \int d \mu \nu_{s}\left(\frac{I v}{\Omega}\right)^{2} \frac{\partial f_{0}^{(0)}}{\partial \psi}\left(\int d \theta \mathcal{J} \frac{B}{v_{\|}}\right) \\
= & -\frac{3}{2 v_{e j \rho}^{2}} \frac{1}{V^{\prime}} \frac{\partial}{\psi}\left[V^{\prime}\left(\int d^{\beta} v \nu_{s}\left(\frac{I v}{\Omega}\right)^{2} v_{\|\|}^{2} f_{0}^{(0)}\right)\right] \\
& +\frac{3}{2 v_{e f j}^{2}}\left\langle\int d^{\beta} v v_{s}\left(\frac{I v}{\Omega_{0}}\right)^{2} v_{\|}^{2} f_{0}^{(0)} \frac{\partial}{\partial \psi} \ln \left(\nu_{s} \mathcal{J} B\left|v_{\|}\right|\right)\right\rangle .
\end{aligned}
$$

The rest of the calculation of the energy transport is parallel to the calculation of the particle transport. After some straightforward algebra, we obtain

$$
\begin{aligned}
\mathcal{Q}= & \mathcal{Q}_{d}+\mathcal{Q}_{e} \\
\frac{\mathcal{Q}_{d}}{T_{e f f}}= & -\frac{15 n_{t}}{T_{s}}\left(\frac{I v_{e f f}}{\Omega_{0}}\right)^{2}\left\{\frac{B_{R}-B}{B_{R}}\right\}_{b}\left[\frac{1}{2 n_{t}} \frac{\partial n_{t}}{\partial \psi}+\frac{1}{T_{e f f}} \frac{\partial T_{e f f}}{\partial \psi}\right], \\
\frac{\mathcal{Q}_{c}}{T_{e f f}}= & -\frac{15 n_{t}}{T_{s}}\left(\frac{I v_{e f f}}{\Omega_{0}}\right)^{2}\left\{\frac{B_{n}-B}{B_{R}}\right\}_{b}\left[\frac{3}{2 T_{e}} \frac{\partial T_{e}}{\partial \psi}-\frac{1}{n_{e}} \frac{\partial n_{e}}{\partial \psi}-\frac{1}{q} \frac{\partial q}{\partial \psi}\right. \\
& \left.+\frac{1}{2} \frac{\partial}{\partial \psi} \ln \left\{\frac{B_{R}-B}{B_{R}}\right\}_{b}\right] \\
& -\frac{45 n_{t}}{4 \tau_{s}}\left(\frac{I v_{e f f}}{\Omega_{0}}\right)^{2}\left\{\frac{\partial \ln B}{\partial \psi}\right\}_{b},
\end{aligned}
$$

where we have separated $\mathcal{Q}$ into two components; $Q_{d}$ the diffusive component and $\mathcal{Q}_{c}$ the convective component. The pitch-angle contribution is neglected here because, unlike the particle flux case, the diffusive terms appear in both slowing-down and pitch-angle scattering contributions. And the slowing-down contribution is always bigger in our ordering where the minority ions are so energetic that $\tau_{s} / \tau_{t}<r / R$ is true, as explained earlier. We can see from Eq. (49) that the diffusive components of $\mathcal{Q}$ are radially outward in the normal case. 


\section{Discussions}

We have studied the neoclassical kinetic behavior of the energetic minority tail ions generated by strong ICRF heating at the fundamental harmonic frequency, and obtained simple kinetic expressions for the radial transport coefficients of the tail minority ions. It is found that the radial particle transport by the Coulomb slowing-down collisions on the background electrons is purely convective. The only diffusive particle transport of the tait ions is from the weak pitch angle scattering on the background ions and is usually smaller than that of convective particle transport. On the other hand, the radial energy transport by Coulomb slowing-down scattering has both diffusive and convective terms, due the spread of the energy absorbed by the particles from the RF waves to the background electrons during the banana motions.

The rate of radial particle transport is small, of order one banana width in a slowing-down time. But the rate of energy transport is larger due to the non-Maxwellian nature of the tail distribution function, and the tail ions can radially lose non-negligible amounts of their energy before they are slowed down by collisions with the electrons. As discussed in the introduction, it is probably important to include both collision-driven transport of the present work and the RF-driven transport of Ref. 5 in the analysis of ICRF heating cases like in JET ${ }^{6.7}$ where the RF-power profile is so well focussed and the tail becomes so energetic that the banana widths of the tail jons become a significant fraction of the RF-power profile width. 
Since the direct effect of ICRF waves on radial transport is absent in the present analyses (due to the assumption that $k_{\|}=0$ ), only Coulomb collisions are responsible for the transport and thus the total particle transport must be automatically ambipular. ${ }^{10}$ For the case when $k_{\|} \neq 0$, there will be some additional transport directly driven by the ICRF waves as cliscussed in Ref. 5.

Our expression for the particle flux $\Gamma$ and the energy flux $\mathcal{Q}$, a. presented in Eqs. $(46,47,49$, and 50), are valid for a large-aspect-ratio, low- $\beta$ tokamak with arbitrary flux surface shapes. For a large-aspect-ratio tokamak with circular concentric flux surfaces, using the relations $B \propto 1 / R \propto 1 /\left(R_{o}+\right.$ $r \cos \theta), \partial \psi / \partial r=r B / q$, and $\cos \theta \simeq 1-\theta^{2} / 2$ valid in the deeply trapped particle limit, we can obtain the following approximation:

$$
\begin{aligned}
&\left\{\frac{B_{R}-B}{B_{R}}\right\}_{b} \simeq \frac{1}{2}\left(\frac{B_{R}-B_{\min }}{B_{R}}\right) \simeq \frac{r}{2 R_{o}}\left(1-\cos \theta_{R}\right), \\
&\left\{\frac{\partial \ln B}{\partial \psi}\right\}_{b} \simeq-\frac{q}{B r R}\{\cos \theta\}_{b},
\end{aligned}
$$

where $B_{\min }$ is the minimum value of $B$ on the flux surface corresponding to $\theta=0$ and $R_{0}$ is the value of $R$ at the magnetic axis. And thus, for a large-aspect-ratio tokamak with concentric circular flux surfaces, we can transform our results as follows:

$$
\begin{aligned}
\nabla \cdot \vec{\Gamma}= & \frac{1}{r} \frac{\partial}{\partial r}\left(r \Gamma^{(r)}\right), \\
\Gamma^{(r)}= & -\frac{3}{2} \frac{n_{t}}{T_{s}} \frac{r}{R}\left(\frac{v_{e f f}}{\Omega_{\theta 0}}\right)^{2}\left(1-\cos \theta_{R}\right)\left[\frac{3}{2} \frac{\partial \ln T_{e}}{\partial r}-\frac{\partial \ln n_{e}}{\partial r}-\frac{\partial \ln g}{\partial r}\right] \\
& +\frac{3}{2} \frac{n_{t}}{\tau_{s}} \frac{1}{R}\left(\frac{v_{e f f}}{\Omega_{\theta 0}}\right)^{2}\{\cos \theta\}_{b}
\end{aligned}
$$




$$
-\frac{1}{\sqrt{2 \pi}} \frac{n_{t}}{\tau_{t i}}\left(\frac{v_{e f f}}{\Omega_{\theta 0}}\right)^{2}\left[\frac{\partial \ln n_{t}}{\partial r}-\frac{1}{2} \frac{\partial \ln T_{e f f}}{\partial r}+\frac{\partial \ln n_{i}}{\partial r}+\frac{\partial \ln q}{\partial r}\right],
$$

where $\Omega_{90}=Z_{t} e B_{\theta 0} / M_{t} c$ is the poloidal gyrofrequency averaged over the flux surface and $\{\cos \theta\}_{b} \simeq 1-\theta_{R}^{2} / 4$ can be used in the deeply trapped limit $\theta \ll 1$. Likewise, the energy balance equation in circular geometry is

$$
\begin{aligned}
\nabla \cdot \overrightarrow{\mathcal{Q}}= & \frac{1}{r} \frac{\partial}{\partial r}\left(r \mathcal{Q}^{(r)}\right) \\
\frac{\mathcal{Q}^{(r)}}{T_{e f f}}= & -\frac{15}{2} \frac{n_{t}}{\tau_{s}} \frac{r}{R}\left(\frac{v_{e f f}}{\Omega_{\theta 0}}\right)^{2}\left(1-\cos \theta_{R}\right)\left[\frac{\partial \ln T_{e f f}}{\partial r}\right. \\
& \left.+\frac{1}{2} \frac{\partial \ln n_{t}}{\partial r}+\frac{3}{2} \frac{\partial \ln T_{e}}{\partial r}-\frac{\partial \ln n_{e}}{\partial r}-\frac{\partial \ln q}{\partial r}\right] \\
& +\frac{45}{4} \frac{n_{t}}{\tau_{s}} \frac{1}{R}\left(\frac{v_{e f f}}{\Omega_{00}}\right)^{2}\left[\frac{1}{3\left(1-\cos \theta_{R}\right)}+\{\cos \theta\}_{b}\right] .
\end{aligned}
$$

The rate of radial particle flow is found to be about one banana width in a slowing-down time. Thus, the loss of fast particles from the central plasma region (which is over several banana widths in radial thickness) is small within a slowing-down time. But the rate of radial energy transport is much faster than a banana width in a slowing-down time. Hence the radial transport processes discussed here should be included in the detailed modeling of the main-plasma heating by the tail ions, which occurs in a slowing-down time scale, and in the analysis of the experimental tail-ion data.

In the calculation of the present neoclassical transport rate, a simple (but realistic) anisotropic tail distribution function has been assumed by taking the limit of small pitch angle spread. The present model distrilyution 
function reduces to the isotropic model solution of Ref. 8 when integrated over pitci angle. Different models of anisotropic tail distribution functions, like those given in Refs. 16 and 18 may yield somewhat different results. However, since all the information we need from the lowest order tail distribution function is a few velocity moments, it is expected that all the essential features of our diffusion coefficients are unchanged, only the numerical coefficients may change a little. We have actually evaluated the differences in the transport coefficients and found that the present model distribution function yielded a little larger coefficient value than other models quoted above did. Thus we may regard the present transport rate as an upper bound to the neoclassical loss of fast ions.

The present analyses may not be valid in the transport estimation of another possibly important class of ICRF-driven minority ions, which is the lower energy tail ions near the critical ion-electron slowing-down energy. In this case, the Coulomb pitch angle scattering will be as strong as the energy scattering and thus the localization of the tail ions near the resonance pitch angle is not possible. Thus, more elaborate analyses of the tail ion hinetics in connection with the passing-trapped transition become necessary.

\section{Acknowledgment}

This work was supported by U.S. Department of Energy under the contract numbers DE-FG02-86-ER53223 and DE-AC02-76CH03073. 


\section{References}

${ }^{1} \mathrm{~J}$. R. Wilson, and PLT Group, in Radiofrequency Plasma Heating, Sixth Topical Conference, Callaway Gardens, GA, 1985 (American Institute of Physics, New York, 1985), p. 28.

${ }^{2}$ D.F.H. Start, V.P .Bhatnager, D.A. Boyd, M. Bures, D.J. Campbell, J.P. Christiansen, P.L. Colestock, J.G. Cordey, W. Core, G.A. Cottrell. L.G. Eriksson, M.P. Evrard, T. Hellsten, J. Jacquinot, O.N. Jarvis, S. Kissel, S. Knowlton, H. Lean, P.J. Lomas, C. Lowry, A.L. McCarthy, P. Nielsen, J. O'Rourke, G. Sadler, A. Tanga, P.R. Thomas, K. Thomsen, B. Tubbing, P. van Belle, and J.A.Wesson, in Proceedings of 12th Inter. rational Conference on Plasma Physics and Controlled Nuclear Fusion Research (IAEA, Vienna, 1989), Vol. 1, p. 593.

${ }^{3}$ J.R. Wilson, M.G. Bell, A. Cavallo, P.L. Colestock, W. Dorland, W. Gardner, G.J. Greene, G. Hammett, R. Hawryluk, H. Hendel, D. Hoffman, J.C. Hosea, K. Jaehnig, F. Jobes, R. Kaita, C. Kieras-Phillips, A. Lysojvan, D. Mansfield, S. Medley, D. Mueiler, K. Owens, D. Smithe. J.E. Stevens, D. Swain, G. Tait, G. Taylor, M. Ulrickson, K.L. Wong. S. Zweben, and the TFTR group, in Proceedings of 12th International Conference on Plasma Physics and Controlled Nuclear Fusion Research (IAEA, Vienna,1989), Vol. 1, p. 691.

${ }^{4}$ C. S. Chang, Phys. Fluids 28, 3598(1985).

${ }^{5}$ Liu Chen, J. Vaclavik, and G. W. Hammett, Nucl. Fusion 28. 389(198S). 
${ }^{6}$ T. Hellsten, Plasma Phys, and Contr. Fusion 31,1391 (1989).

${ }^{i}$ W.G.F. Core, Nucl. Fusion 29, 1101(1089).

${ }^{8}$ T. H. Stix, Nucl. Fusion $15,737(1975)$.

${ }^{9}$ R. Kaita et al., Nucl. Fusion 23, 1089(1983); D. M. Manos et al., J. Nucl. Materials 120, 319(1984); M. A. Rothman, R. M. Sinclair, I. G. Brown, and J. C. Hosea, Phys. Fluids 12, 2211(1969).

${ }^{10}$ F. L. Hinton and R. D. Hazeltine, Rev. Mod. Phys. $48,239(1976)$.

${ }^{11}$ A. A. Ware, Phys. Rev. Lett. 25, 916(1970).

${ }^{12}$ A. A. Galeev, Sov. Phys. 32, 752(1971).

${ }^{13}$ A. A. Vedenov, E. P. Velikhov, and R. Z. Sagdeev, Nucl. Fusion, Suppl. 2, 465(1962); W. E. Drummond and D. Pines, Nucl. Fusion, Suppl. 3. $1049(1962)$.

${ }^{1+}$ T.H. Stix, Plasma Physics 14, 367(19i1).

${ }^{15} \mathrm{C}$. F. Kennel and F. Engelmann, Phys. Fluids 9, 2377(1966).

${ }^{16}$ G. W. Hammett, Ph. D. Thesis, Department of Astrophysical Sciences. Princeton University, 1986.

${ }^{17} G$. Kerbel and M. McCoy, Phys. Fluids 28, 3629(1985).

${ }^{18} \mathrm{C}$. S. Chang and P. Colestock, Phys. Fluids (to be published 1. 


\section{Figures}

FIG. 1. Schematic diagram of a fast ion's banana motion. It is heated at the banana tips and cooled during the banana motion. The verticle dashed line represents the resonance surface where the particle is taking energy from the RF wave.

FIG. 2. Convective radial motion of a fast ion due to the asymmetric amount of drag between the outside and inside banana motions. 
$\# 89 \times 0289$

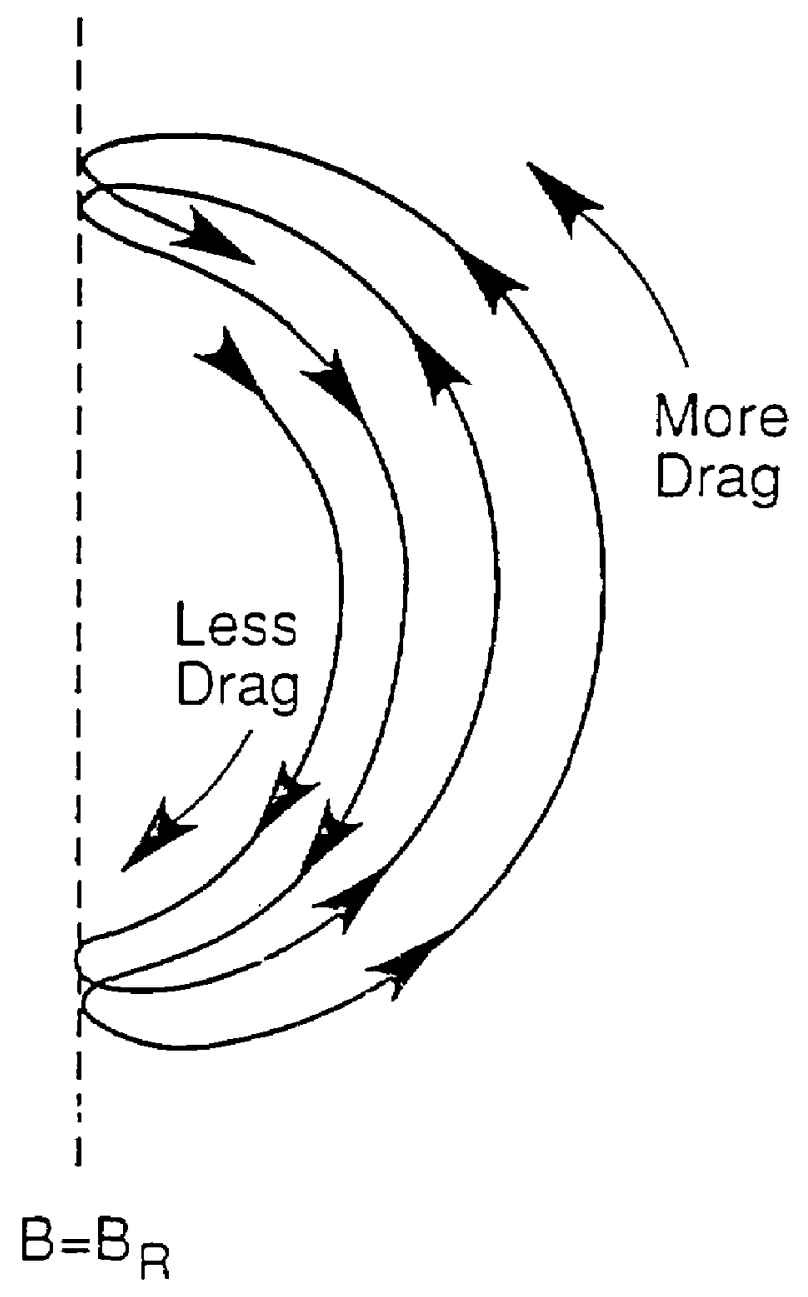

FIG. 1 


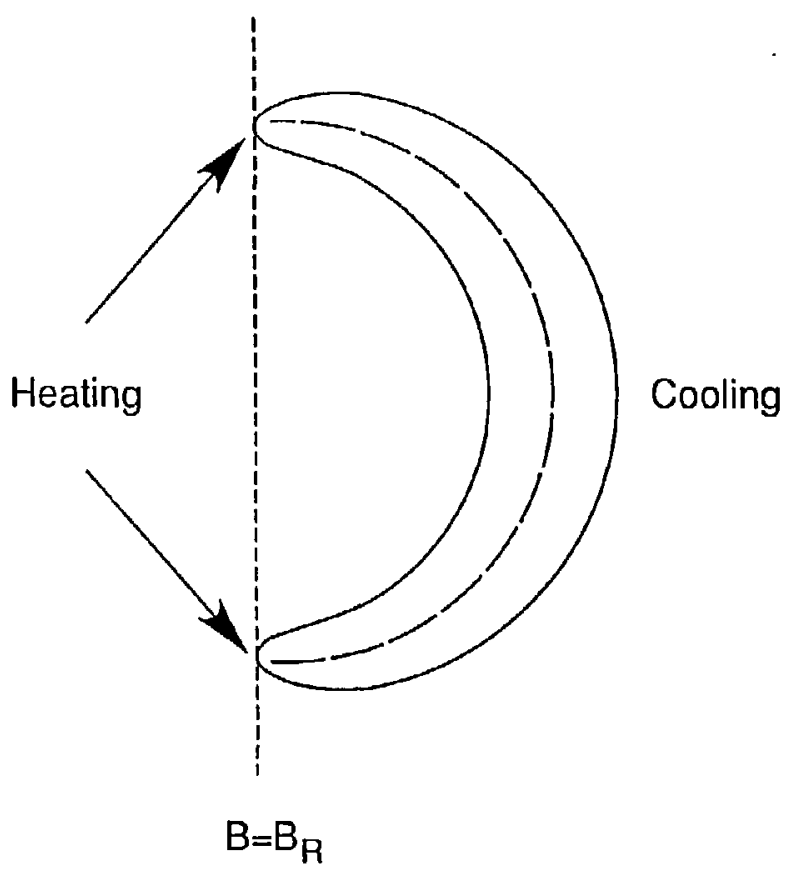

